\title{
Comparison of the Models for Multiscale Elastohydrodynamic Lubrication in a Line Contact
}

\author{
W. $\operatorname{Lin}^{1,2}, \mathrm{~J}^{\mathrm{Li}^{2}}$ and Y. Zhang ${ }^{2 \dagger}$ \\ 1. School of Mechanics Technology, Wuxi Institute of Technology, Wuxi, Jiangsu Province, China \\ 2 College of Mechanical Engineering, Changzhou University, Changzhou, Jiangsu Province, China \\ †Corresponding Author Email: engmech1@sina.com
}

(Received June 28, 2021; accepted October 31, 2021)

\begin{abstract}
Two models are compared for calculating the surface separation in a multiscale elastohydrodynamic lubricated line contact for the same operating conditions. In the studied line contact, the surface separation is very low so that the effect of the adsorbed boundary layer is significant. Model I principally takes the continuum fluid film as intervening between the two adsorbed boundary layers. Model II takes the continuous phase transition both along the flow direction and across the whole surface separation; in this model, in the Hertzian contact zone there is only the adsorbed boundary layer, while in most of the inlet zone there is only the continuum fluid film (by neglecting the adsorbed boundary layer). The analytical results show that for the same case these two models give the close surface separations. The equivalence of these two models is shown.
\end{abstract}

Keywords: Contact; Hydrodynamics; Mixed rheology; Model; Multiscale.

\section{NOMENCLATURE}

\begin{tabular}{|c|c|c|c|}
\hline$l_{0}, a_{1}, a$ & $\begin{array}{ll}I_{2} & \text { constant respectively } \\
\text { half Hertzian contact width }\end{array}$ & $h_{c}$ & $\begin{array}{l}\text { dimensional film thickness of the } \\
\text { continuum fluid at the contact center }\end{array}$ \\
\hline$b$ & half Hertzian contact width & & continuum fluid at the contact center \\
\hline$C_{y, 1}$ & $\eta_{b f, 1}^{\text {eff }} / \eta$ & $n_{c r, b f, 1}$ & $\begin{array}{l}\text { critical thickness for characterizing the } \\
\text { rheological properties of the adsorbed }\end{array}$ \\
\hline$y, 2$ & $\eta_{b f, 2}^{\text {eff }} / \eta$ & & layer in Model I \\
\hline$D$ & fluid molecule diameter & $h_{c r, b f, 2}$ & critical thickness for characterizing the \\
\hline$E_{a}, E_{b}$ & $\begin{array}{l}\text { Young's moduli of the upper and lower } \\
\text { contact surfaces respectively }\end{array}$ & & $\begin{array}{l}\text { rheological properties of the boundary } \\
\text { layer in the Hertzian contact zone }\end{array}$ \\
\hline$E_{v}$ & $2\left(1-v_{a}^{2}\right) / E_{a}+2\left(1-v_{b}^{2}\right) / E_{b}$ & $h_{c, t o t}$ & dimensional surface separation at the \\
\hline$G$ & $\alpha E_{v}$ & & ntact center \\
\hline$H$ & $h / R$ & $h_{c, t o t, N}$ & surface separation \\
\hline$H_{b f}$ & $h_{b f} / R$ & $h_{\text {ot }}$ & $\begin{array}{l}\text { contact center in Fig.1(c) (in Model II) } \\
\text { dimensional surface separation }\end{array}$ \\
\hline $\bar{H}_{b f}$ & $h_{b f} / h_{c r, b f, 1}$ & $k$ & parameter \\
\hline$H_{c}$ & $\begin{array}{l}\text { dimensionless film thickness of the } \\
\text { continuum fluid }\end{array}$ & $m$ & $\begin{array}{l}\text { exponential index for local viscosity } \\
\text { ratio within the adsorbed layer }\end{array}$ \\
\hline$H_{\text {tot }}$ & $h_{t o t} / R$ & $n$ & $\begin{array}{l}\text { equivalent number of the molecules fluid } \\
\text { across the layer thickness }\end{array}$ \\
\hline $\bar{H}_{t o t}$ & $h_{\text {tot }} / h_{c r, b f, 2}$ & $p$ & pressure \\
\hline$H_{c, t o t}$ & $h_{c, t o t} / R$ & $P$ & $p / p_{h}$ \\
\hline$H_{c, t o t, N}$ & $h_{c, t o t, N} / R$ & $p_{h}$ & maximum Hertzian contact pressure \\
\hline$h_{b f}$ & thickness of the adsorbed layer & $q_{0}$ & $\Delta_{j+1} / \Delta_{j}(>1)$ \\
\hline${ }_{j-1}$ & viscosity & $q_{v, b f, A}$ & $\begin{array}{l}q_{v, b f, B} \text { volume flow rates per unit } \\
\text { contact length of the upp }\end{array}$ \\
\hline
\end{tabular}




\author{
the $j^{\text {th }}$ and $(j-1)^{\text {th }}$ fluid molecules across \\ the layer thickness \\ $v_{a} \quad$ Poisson's ratio of the upper contact surface \\ $v_{b} \quad$ Poisson's ratio of the lower contact surface \\ $\lambda \quad b /(4 R)$ \\ $\lambda_{b f} \quad h_{b f} / h$ \\ $\lambda_{b f, e} \quad h_{b f} /\left(k h_{c}\right)$ \\ $\alpha \quad$ fluid viscosity-pressure index \\ $\eta \quad$ fluid bulk viscosity \\ $\eta_{a} \quad$ fluid bulk viscosity at ambient pressure \\ $\eta_{b f, 1}^{e f f}$ effective viscosity of the physical adsorbed \\ layer \\ $\eta_{b f, 2}^{e f f}$ effective viscosity of the boundary layer \\ across the whole surface separation \\ in the Hertzian contact zone \\ $\Delta_{n-2}$ separation between the neighboring fluid \\ molecules across the layer thickness just \\ on the adsorbed layer-fluid interface \\ $\Delta_{j-1} \quad$ separation between the $j^{\text {th }}$ and $(j-1)^{h}$ fluid \\ molecules across the layer thickness
}

\author{
lower adsorbed layers respectively \\ $q_{v, h f} \quad$ volume flow rate per unit contact length \\ of the intermediate continuum fluid \\ $R \quad R_{a} R_{b} /\left(R_{a}+R_{b}\right)$ \\ $R_{a}, R_{b}$ curvature radii of the upper and lower \\ contact surfaces respectively \\ $S \quad$ parameter describing the non-continuum \\ effect of the boundary layer confined in \\ the whole surface separation \\ $U$ dimensionless rolling speed \\ $u_{a}, u_{b}$ circumferential speeds of the upper and \\ lower contact surfaces respectively \\ w load per unit contact length carried by the \\ contact \\ W dimensionless load \\ $x \quad$ coordinate along the contact \\ $X \quad x / b$ \\ $x_{0} \quad$ dimensionless $x$ coordinate of the boundary \\ between the intermediate continuum fluid \\ film area and the continuum fluid film \\ vanishing area in the inlet zone \\ $\Delta x \quad$ separation between the neighboring fluid \\ molecules in the flow direction in the \\ adsorbed layer
}

\section{INTRODUCTION}

Line contact is an important contact mode on mechanical elements such as gears, cams and roller bearings (Kalker 1972). Elastohydrodynamic lubrication in a line contact was classically described as continuum lubrication (Grubin 1949; Pinkus and Sternlicht 1961). However, when carrying a heavy load, the surface separation in a lubricated concentrated contact will be very low and comparable with the thickness of the boundary layer physically adsorbed to the contact surface (Begelinger and Gee de 1974, 1976). For this case, it has been found that the boundary layer effect should be considered and it can greatly increase the lubricating film thickness for a given load (Zhang 2020, 2021b).

Multiscale elastohydrodynamic lubrication has been proposed for line contacts (Zhang 2020, 2021b). In this theory, there are two adsorbed boundary layers respectively on the contact surfaces, intermediate between them there is a continuum fluid film; three equations are required respectively for describing the flow in the adsorbed layers and in the intermediate continuum fluid. When the surface separation is critically low, in the Hertzian zone of a line contact there is only the adsorbed layer, but in most of the inlet zone there are the adsorbed layers and the intermediate continuum fluid (Zhang 2020). The multiscale elastohydrodynamic lubrication theory has been developed for this line contact (Zhang 2020, 2021b).

In the earlier researches (Zhang et al. 2003, Zhang and Lu 2003), another model for modeling the above mentioned multiscale elastohydrodynamic lubrication was proposed. In that model, in the Hertzian zone there was only the adsorbed boundary layer, the rheological properties of which were continuously transited both along the flow direction and across the surface separation; the effective viscosity and the average density of the adsorbed layer across the surface separation were used for describing the adsorbed layer flow; in the other contact areas there were only continuum fluid films, the flow of which was described by the continuum fluid model. Plentiful results have been obtained based on that model. That earlier model was actually developed under the popular misleading understanding at that time that in a hydrodynamic lubricated contact except the local adsorbed boundary layer there is only the continuum fluid film (Johnston et al. 1991). The phenomenon most challenging to this concept is the multiscale hydrodynamic lubrication regime in which both the adsorbed boundary layer and the intermediate continuum fluid film are present in the whole contact. Chan and Horn (1985) may be right to assume a boundary layer on the contact surface to explain the experimentally observed anomalous drainage force in the hydrodynamic lubricated concentrated contact with nanoscale surface separations but not propose the new lubrication terminology for the observed anomaly.

It is now necessary to evaluate these two models when simulating hydrodynamic lubrication with ultra low surface clearances. Such a research will help to solve the issue of the model applicability in the following time. The present paper just shows this research.

The studied object here is smooth steel line contacts, which are hydrophilic or oilphilic, lubricated by common liquids. In this case, air should not be entrained between the solid surface and the adsorbed layer so that no interfacial 
slippage occurs (Blake and Haynes 1969; Karim and Kavehpour 2015; Karim et al. 2016, 2018, 2021b; Petrov and Petrov 1992). Therefore, in this work, the lubricating liquid is assumed to completely spread over the solid surface.

\section{MODEL CLARIFICATION}

\subsection{Model I: Continuum fluid film intervening between two adsorbed boundary layers}

Figures 1(a) and (b) show Model I for multiscale elastohydrodynamic lubrication in the line contact where the continuum fluid film intervenes between the two adsorbed boundary layers (Zhang 2020, 2021b). The adsorbed boundary layer is very thin and its flow is essentially nanoscale and non-continuum, while the intermediate continuum fluid flow is macroscale and can be described by the classical continuum fluid model (Atkas and Aluru 2002; Yen et al. 2007). These two flows are on largely different scales with qualitatively different flow regimes and the corresponding multiscale flow modeling should handle the boundary condition between the adsorbed layer and the continuum fluid.

When the surface separation is relatively high, between the two adsorbed boundary layers there will always be the intervening continuum fluid film in the whole contact, as shown in Fig. 1(a). When the surface separation is ultra low, there will be only the adsorbed boundary layer in the Hertzian contact zone, while in most of the inlet zone there will still be the continuum fluid film intervening between the two adsorbed boundary layers, as shown in Fig.1(b). For Fig. 1(a), three closed-form explicit flow equations have been derived respectively for the flows in the two adsorbed boundary layers and the flow in the intermediate continuum fluid (Zhang 2020). Detailed analytical results for the elastohydrodynamic lubricated line contact based on this model have been shown (Zhang 2021b) when the two adsorbed layers were assumed as identical. Here are only briefly repeated the necessary contents from those studies.

The volume flow rates per unit contact length of the upper and lower adsorbed boundary layers in Figs. 1(a) and (b) are respectively (Zhang 2020, 2021b):

$$
\begin{gathered}
q_{v, b f, A}=u_{a} h_{b f}+\frac{u_{b}-u_{a}}{2} \\
\frac{\varepsilon h_{b f} \lambda_{b f}}{2 \lambda_{b f}+C_{y, 1}\left(1+\frac{\Delta x}{D}\right)} \\
+\frac{F_{1} h_{b f}^{3}}{12 \eta_{b f, 1}^{e f f}} \frac{\partial p}{\partial x}-\frac{\varepsilon}{1+\frac{\Delta x}{D}} \frac{h_{b f}^{3}}{2 \eta_{b f, 1}^{e f f}} \frac{\partial p}{\partial x} \\
\cdot\left(1+\frac{1}{2 \lambda_{b f}}-\frac{q_{0}-q_{0}^{n}}{q_{0}^{n-1}-q_{0}^{n}} \frac{\Delta_{n-2}}{h_{b f}}\right)
\end{gathered}
$$

and

$$
\begin{gathered}
q_{v, b f, B}=u_{b} h_{b f}+\frac{u_{a}-u_{b}}{2} \\
\cdot \frac{\varepsilon h_{b f} \lambda_{b f}}{2 \lambda_{b f}+C_{y, 1}\left(1+\frac{\Delta x}{D}\right)} \\
+\frac{F_{1} h_{b f}^{3}}{12 \eta_{b f, 1}^{e f f}} \frac{\partial p}{\partial x}-\frac{\varepsilon}{1+\frac{\Delta x}{D}} \frac{h_{b f}^{3}}{2 \eta_{b f, 1}^{e f f}} \frac{\partial p}{\partial x} \\
\cdot\left(1+\frac{1}{2 \lambda_{b f}}-\frac{q_{0}-q_{0}^{n}}{q_{0}^{n-1}-q_{0}^{n}} \frac{\Delta_{n-2}}{h_{b f}}\right)
\end{gathered}
$$

where $u_{a}$ and $u_{b}$ are respectively the circumferential speeds of the upper and lower contact surfaces, $h_{b f}$ is the thickness of the adsorbed layer, $p$ is the hydrodynamic pressure, $X$ is the coordinate along the contact as shown in Fig.1, $D$ is the fluid molecule diameter, $\lambda_{b f}=h_{b f} / h \quad(h$ is the thickness of the intermediate continuum fluid film), $\eta_{b f, 1}^{\text {eff }}$ is the effective viscosity of the physical adsorbed layer and $\eta_{b f, 1}^{\text {eff }}=D h_{b f} /\left[(n-1)\left(D+\Delta_{x}\right)\left(\Delta_{l} / \eta_{\text {line,l }}\right)_{a v r, n-1}\right]$, $C_{y, 1}=\eta_{b f, 1}^{e f f} / \eta, \eta$ is the fluid bulk viscosity, $\Delta x$ is the separation between the neighboring fluid molecules in the flow direction in the adsorbed layer, $q_{0}=\Delta_{j+1} / \Delta_{j} \quad\left(\Delta_{j}\right.$ is the separation between the $(j+1)^{\text {th }}$ and $j^{\text {th }}$ fluid molecules across the layer thickness) and $q_{0}$ is constant, $\eta_{\text {line } j} / \eta_{\text {line } j+1}=q_{0}^{m} \quad, \quad \varepsilon=(2 D I+I I) /$ $\left[h_{b f}(n-1)\left(\Delta_{l} / \eta_{\text {line }, l}\right)_{a v r, n-1}\right] \quad, \quad F_{1}=\eta_{b f, 1}^{e f f}$ $\left(12 D^{2} \Psi+6 D \Phi\right) / h_{b f}^{3}, n$ is the equivalent number of the fluid molecules across the layer thickness, and $\Delta_{n-2}$ is the separation between the neighboring fluid molecules across the layer thickness just on the adsorbed layer-fluid interface.

Here, $I=\sum_{i=1}^{n-1} i\left(\Delta_{l} / \eta_{\text {line }, l}\right)_{a v r, i}$,

$\Psi=\sum_{i=1}^{n-1} i\left(l \Delta_{l-1} / \eta_{\text {line }, l-1}\right)_{a v r, i}$,

$I I=\sum_{i=0}^{n-2}\left[i\left(\Delta_{l} / \eta_{\text {line }, l}\right)_{a v r, i}+(i+1)\left(\Delta_{l} / \eta_{\text {line }, l}\right)_{a v r, i+1}\right] \Delta_{i}$,

$\left.\Phi=\sum_{i=0}^{n-2} i\left(I \Delta_{-1} / \eta_{\text {ire },-1}\right)_{\text {ar }, j}+(i+1)\left(I \Delta_{-1} / \eta_{\text {ire },-1}\right)_{\text {ar }, i+1}\right] A_{4}$,

$i\left(\Delta_{l} / \eta_{\text {line }, l}\right)_{\text {avr }, i}=\sum_{j=1}^{i} \Delta_{j-1} / \eta_{\text {line }, j-1}$, and

$i\left(l \Delta_{l-1} / \eta_{\text {line }, l-1}\right)_{a v r, i}=\sum_{j=1}^{i} j \Delta_{j-1} / \eta_{\text {line }, j-1} ;$

$\eta_{\text {line }, l-1}$ is the local viscosity between the $j^{\text {th }}$ and $(j-$

$1)^{\text {th }}$ fluid molecules across the layer thickness. 
W. Lin et al. / JAFM, Vol. 15, No. 2, pp. 515-521, 2022.

The volume flow rate per unit contact length of the intermediate continuum fluid is (Zhang 2020, 2021b):

$$
\begin{aligned}
& q_{v, h f}=\frac{u_{a}+u_{b}}{2} h-\frac{h^{3}}{12 \eta} \frac{\partial p}{\partial x} \\
&+ \frac{h^{3}}{\eta_{b f f}^{e f f}} \frac{\partial p}{\partial x}\left[\frac{F_{2} \lambda_{b f}^{2}}{6}-\frac{\lambda_{b f}}{1+\frac{\Delta x}{D}}\right. \\
&\left.\cdot\left(\frac{1}{2}+\lambda_{b f}-\frac{q_{0}-q_{0}^{n}}{q_{0}^{n-1}-q_{0}^{n}} \frac{\Delta_{n-2}}{h}\right)\right]
\end{aligned}
$$

where $F_{2}=6 \eta_{b f, 1}^{e f f} D(n-1)\left(l \Delta_{l-1} / \eta_{\text {line }, l-1}\right)_{a v r, n-1} / h_{b f}^{2}$.

The flow continuity equation for both the contact in Fig.1(a) and the contact in Fig.1(b) for $X<X_{0}$ is:

$$
q_{v, b f, A}+q_{v, b f, B}+q_{v, h f}=\frac{u_{a}+u_{b}}{2} h_{c, t o t}
$$

where $h_{c, t o t}$ is the surface separation in the location where the pressure gradient is vanishing.

Substituting Eqs. (1)-(3) into Eq. (4) gives the following final dimensionless governing equation for both the contact in Fig.1(a) and the contact in Fig.1(b) for $X<X_{0}$ (Zhang 2020, 2021b):

$\frac{1}{4} F\left(\lambda_{b f}\right) H^{3} e^{-P G \lambda} \frac{d P}{d X}=U\left(H-H_{0}\right)$

where $X=x / b \quad, \quad U=\left(u_{a}+u_{b}\right) \eta_{a} /\left(2 E_{v} R\right)$, $P=p / p_{h}, G=\alpha E_{v}, \lambda=b /(4 R), H=h / R$, $H_{0}=H_{c, \text { tot }}-2 H_{b f} \quad, \quad H_{c, \text { tot }}=h_{c, \text { tot }} / R$ , $H_{b f}=h_{b f} / R, R$ is the equivalent curvature radius of the two contact surfaces and:

$$
\begin{gathered}
F\left(\lambda_{b f}\right)=\frac{1}{12}-\frac{1}{C_{y, 1}}\left[\frac{F_{2} \lambda_{b f}^{2}}{6}-\frac{\lambda_{b f}}{1+\frac{\Delta x}{D}}\right. \\
\left.\cdot\left(\frac{1}{2}+\lambda_{b f}-\frac{q_{0}-q_{0}^{n}}{q_{0}^{n-1}-q_{0}^{n}} \frac{\Delta_{n-2} \lambda_{b f}}{h_{b f}}\right)\right] \\
-\frac{F_{1} \lambda_{b f}^{3}}{6 C_{y, 1}}+\frac{\varepsilon \lambda_{b f}^{3}}{C_{y, 1}\left(1+\frac{\Delta x}{D}\right)} \\
\cdot\left(1+\frac{1}{2 \lambda_{b f}}-\frac{q_{0}-q_{0}^{n}}{q_{0}^{n-1}-q_{0}^{n}} \frac{\Delta_{n-2}}{h_{b f}}\right)
\end{gathered}
$$

Here $E_{v}$ is the equivalent Young's modulus of the two contact surfaces, $p_{h}$ is the maximum Hertzian contact pressure, $b$ is the half Hertzian contact width, $\eta_{a}$ is the fluid bulk viscosity in the ambient condition, and $\alpha$ is the fluid viscosity-pressure index.

Integrating Eq.(5) for the contact in Fig.1(a) gives the following equation for solving the dimensionless intermediate continuum fluid film thickness $H_{c}$ (Zhang 2021b):

$$
\begin{aligned}
& -0.2041\left(\lg \frac{W}{H_{c}}\right)^{2}+0.8876 \lg \frac{W}{H_{c}} \\
& +\lg \left[\frac{4 G U}{\pi F\left(\lambda_{b f, e}\right)}\left(\frac{8}{\pi}\right)^{1 / 2}\right]-\frac{3}{2} \lg W \\
& -1.2725=0, \\
& \text { for } 0.01 \leq \frac{W}{H_{c}} \leq 200
\end{aligned}
$$

where $H_{c}=h_{c} / R \quad, \quad W=w /\left(E_{v} R\right) \quad, \quad$ and $\lambda_{b f, e}=h_{b f} /\left(k h_{c}\right)(1 \leq k<+\infty)$. It was shown that the value of $k$ can be taken as unity (Zhang 2021b). Once $H_{c}$ is solved, the dimensionless surface separation at the contact center in Fig. 1(a) is: $H_{c, \text { tot }}=H_{c}+2 H_{b f}$.

Integrating Eq.(5) for $-\infty<X<X_{0}$ in Fig.1(b) gives that:

$$
\begin{aligned}
J_{2} & =\int_{-\infty}^{X_{0}} \frac{H-H_{0}}{H^{3} F\left(\lambda_{b f}\right)} d X \\
& =\int_{0}^{P\left(X_{0}\right)} \frac{e^{-P G \lambda}}{4 U} d P=\frac{1-e^{-P\left(X_{0}\right) G \lambda}}{4 U G \lambda}
\end{aligned}
$$

The flow of the boundary layer in the Hertzian contact zone in Fig.1(b) is described by the nanoscale flow equation as follows (Zhang 2016, 2020):

$\frac{S H_{t o t}^{3}}{48 C_{y, 2}} e^{-P G \lambda} \frac{d P}{d X}=U\left(H_{c, t o t}-H_{t o t}\right)$

where $H_{\text {tot }}=h_{\text {tot }} / R, C_{y, 2}=\eta_{b f, 2}^{\text {eff }} / \eta, \eta_{b f, 2}^{\text {eff }}$ is the effective viscosity of the boundary layer across the whole surface separation in the Hertzian zone, and $S$ is the parameter describing the noncontinuum effect of the boundary layer across the whole surface separation.

Integrating Eq. (9) for $X_{0} \leq X \leq-1$ in Fig. 1(b) gives that (Zhang 2020):

$P\left(X_{0}\right)=-\frac{\ln \left(-48 U G \lambda J_{3}\right)}{G \lambda}$

where $J_{3}=\int_{X_{0}}^{-1} C_{y, 2}\left(H_{t o t}-H_{c, t o t}\right) d X /\left(S H_{\text {tot }}^{3}\right)$.

Solving the coupled Eqs. (8) and (10) gives that (Zhang 2020):

$J_{2}-12 J_{3}=\frac{1}{4 U G \lambda}=\frac{1}{U G}\left(\frac{\pi}{8 W}\right)^{1 / 2}$

The surface separation $H_{c, t o t}$ in the contact in Fig. 1(b) is solved from Eq. (11). 


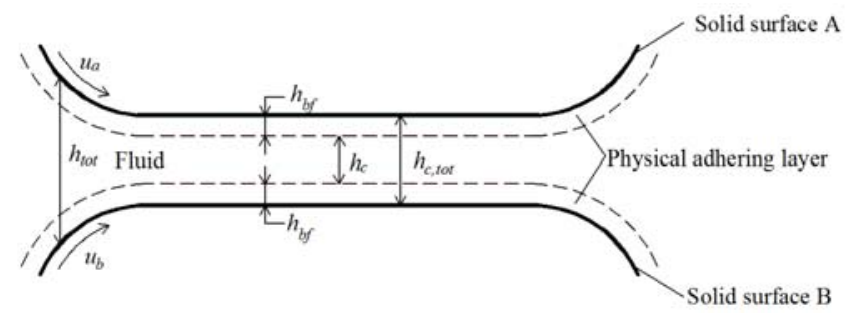

(a)

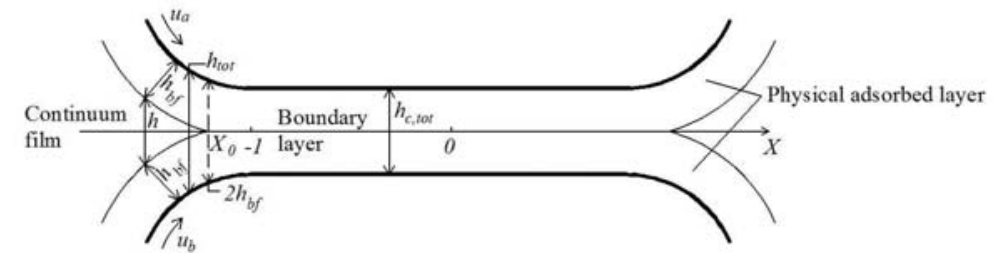

(b)

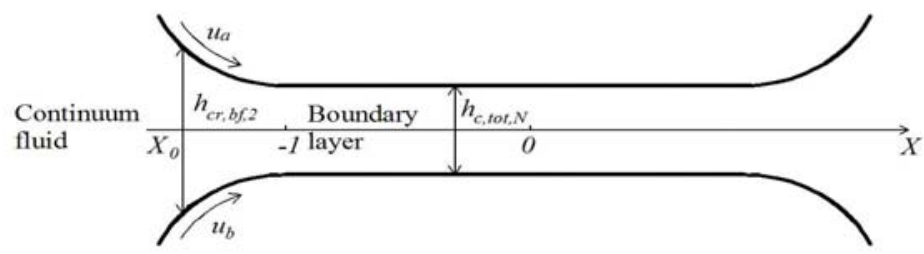

(c)

Fig. 1. Two models for the multiscale elastohydrodynamic lubrication in the line contact (Zhang 2020, 2021b). (a) and (b): Model I, the continuum fluid film intervening between the two adsorbed layers; (c) Model II: Across the surface separation, there is no mixture of the continuum fluid film and the adsorbed layer.

\subsection{Model II: Model of the contact in Fig.1(c)}

The model assumes that in the contact in Fig. 1(c), in the inlet area where $X<X_{0}$ there is only the continuum fluid film, which is described by the Newtonian fluid model. By taking $h_{b f}=0$, Eq.(8) is also valid for $X<X_{0}$ for the contact in Fig. 1(c).

This model assumes that in the inlet area where $X_{0} \leq X \leq-1$, there is only the adsorbed layer across the whole surface separation. The governing equation for this adsorbed layer is still Eq. (10). The dimensionless surface separation $H_{c, t o t, N}$ in the contact in Fig. 1(c) is still solved from Eq. (11) if taking $h_{b f}=0$.

The dimensionless coordinate $X_{0}$ on the boundary between the continuum fluid film area and the pure boundary layer area in the inlet zone in Fig. 1(c) is solved from the following equation:

$$
\begin{gathered}
\frac{h_{c r, b f, 2}}{R}=H_{c, t o t, N}-\frac{4}{\pi}\left\{X_{0}\left(X_{0}{ }^{2}-1\right)^{0.5}\right. \\
\left.+\ln \left[-X_{0}-\left(X_{0}{ }^{2}-1\right)^{0.5}\right]\right\} W
\end{gathered}
$$

\section{ANALYSIS}

The analysis was made for the typical case $G=4500$ and $W=1.0 \mathrm{E}-3$, for a relatively strong fluid-contact interaction. The parameter $C_{y, 1}$ is expressed as (Zhang 2020):

$C_{y, 1}\left(\bar{H}_{b f}\right)=1.8335-\frac{1.4252}{\bar{H}_{b f}}+\frac{0.5917}{\bar{H}_{b f}^{2}}$

where $\bar{H}_{b f}=h_{b f} / h_{c r, b f, 1}$, and $h_{c r, b f, 1}$ is the critical thickness for characterizing the rheological properties of the adsorbed layer and here it is taken as $20 \mathrm{~nm}$.

The parameter $C_{y, 2}$ is formulated as (Zhang 2020):

$C_{y, 2}\left(\bar{H}_{t o t}\right)=1.8335-\frac{1.4252}{\bar{H}_{t o t}}+\frac{0.5917}{\bar{H}_{t o t}^{2}}$

where $\quad \bar{H}_{t o t}=h_{t o t} / h_{c r, b f, 2}, h_{t o t}$ is the surface separation for $x_{0} \leq x \leq-1$, and $h_{c r, b f, 2}$ is the critical thickness for characterizing the rheological properties of the adsorbed boundary layer across the whole surface separation and it is equal to $40 \mathrm{~nm}$. 
W. Lin et al. / JAFM, Vol. 15, No. 2, pp. 515-521, 2022.

The parameter $S$ is formulated as (Zhang 2020):

$$
\begin{aligned}
& S\left(\bar{H}_{\text {tot }}\right)= \\
& \left\{\begin{array}{ccc}
-1 & \text {,for } & \bar{H}_{\text {tot }} \geq 1 \\
{\left[0.4-1.374\left(\bar{H}_{\text {tot }}-0.035\right)^{-0.534}\right]^{-1}} & \text {,for } & 0.035<\bar{H}_{\text {tot }}<1
\end{array}\right.
\end{aligned}
$$

The other input parameter values for the two models were all taken as follows (Zhang 2020, 2021b):

$D=0.5 \mathrm{~nm}, \Delta_{n-2} / D=\Delta x / D=0.15, m=1.5$,

$n=8, q_{0}=1.2$

These values give $h_{b f}=4.32 \mathrm{~nm}$ for both the models.

\section{RESUlts}

Figure 2(a) shows the comparison between the dimensional surface separations respectively calculated from Model I and Model II for $R=0.1 \mathrm{~mm}$ and $R=0.01 \mathrm{~mm}$, when the operating condition is the same. It is shown that these two models give the close results. For low and high dimensionless rolling speeds, the two models match well. For medium rolling speeds, Model II gives the surface separation a bit lower than Model I. Although in itself Model I is more rational, Model II is shown to be compatible with Model I. Both the models give the variations of the dimensional surface separation with the rolling speed matching with the experimental results in a wide rolling speed range. The two models both are of significant interest to the modeling of the related subjects (Chan and Horn 1985; Gohar and Cameron 1963).

Figure 2(b) shows the similar comparisons as in Fig. 2(a) respectively for $R=10 \mathrm{~mm}$ and $R=1 \mathrm{~mm}$. The comparison between Fig. 2(a) and Fig. 2(b) shows that bigger the value of $R$, better the matching of Model I with Model II. For $R=10 \mathrm{~mm}$, the two models give nearly the same results for wide rolling speeds.

\section{CONCLUSIONS}

Two models are introduced for modeling multiscale elastohydrodynamic lubrication in the line contact where the effect of the adsorbed boundary layer on the solid surface is considered. Model I treats the continuum fluid film as intervening between the two adsorbed boundary layers; Model II neglects this intervening but takes the continuous phase transition both across the whole surface separation and in the flow direction, and it divides the lubricated area in the inlet zone into two sub areas where there are respectively the continuum fluid film and the adsorbed boundary layer.

The comparison between these two models was made when calculating the dimensional surface separation in the lubricated line contact for wide rolling speeds. Typical analysis shows the interesting results that these two models are

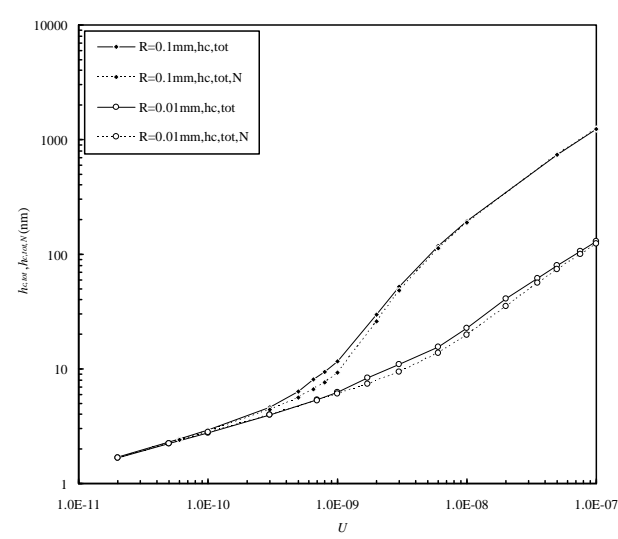

(a)

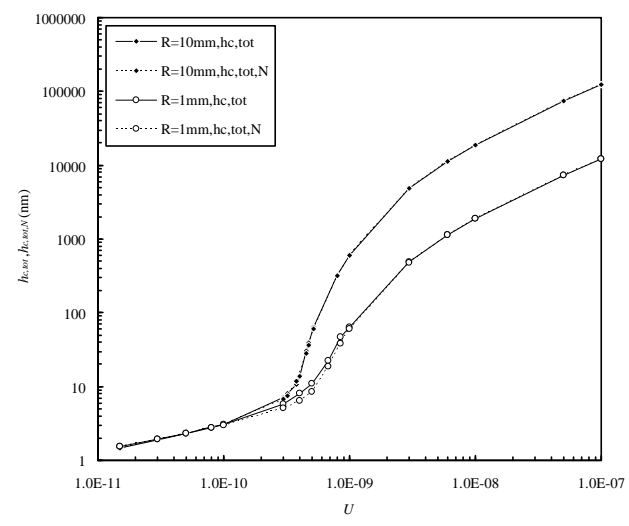

(b)

Fig. 2. Comparison between the dimensional surface separations respectively calculated from Model I and Model II.

altogether matching well. For low and high rolling speeds or for the value of the equivalent curvature radius $R$ of the two contact surfaces on the scale of $10 \mathrm{~mm}$, the two models give nearly the same surface separations for the same operating conditions. When $R$ is as low as on the scales of $0.1 \mathrm{~mm}$ and $0.01 \mathrm{~mm}$, for medium rolling speeds, Model II gives the surface separation a bit lower than Model I. The present study shows the compatibility of these two models in simulating multiscale elastohydrodynamic lu-brication in the line contact.

Model I is physically more reasonable and reliable for the relevant hydrodynamic modeling. The advantage of Model II is its more convenience for use due to ignoring the multiscale hydrodynamic behavior across the surface separation.

\section{REFERENCES}

Atkas, O. and N. R. Aluru (2002). A combined continuum/DSMC technique for multiscale analysis of microfluidic filters. Journal of Computational Physics 178, 342-372.

Blake, T. D. and J. M. Haynes (1969). Kinetics of liquid/liquid displacement. Journal of Colloid 
W. Lin et al. / JAFM, Vol. 15, No. 2, pp. 515-521, 2022.

and Interface Science 30, 421-423.

Begelinger, A. and A. W. J. Gee de (1974). Thin film lubrication of sliding point contacts of AISI 52100 steel. Wear 28, 103-114.

Begelinger, A. and A. W. J. Gee de (1976). On the mechanism of lubricant film failure in sliding concentrated steel contacts. Journal of Tribology 98(4), 575.

Chan, D. Y. C. and R. G. Horn (1985). The drainage of thin liquid films between solid surfaces. Journal of Chemical Physics 83, 5311-5324.

Gohar, R. and A. Cameron (1963). Optical measurement of oil film thickness under elasto-hydrodynamic lubrication. Nature 200, 458-459.

Grubin, A. N. (1949). Fundamentals of the hydrodynamic theory of lubrication of heavily loaded cylindrical surfaces, in Kh. F. Ketova (Ed.). Central Scientific Research Institute for Technology and Mechanical Engineering, D.S.I.R. London Translations, Wellington 337, 115.

Johnston, G. J., R. Wayte and H. A. Spikes (1991). The measurement and study of very thin lubricant films in concentrated contacts. Tribology Transactions 34, 187-194.

Kalker, J. J. (1972). On elastic line contacts. Journal of Applied Mechanics 39, 1125-1132.

Karim, A. M. and H. P. Kavehpour (2015). Dynamics of spreading on ultra-hydrophobic surfaces. Journal of Coating Technology Research 12, 959-964.

Karim, A. M., S. H. Davis and H. P. Kavehpour (2016). Forced versus spontaneous spreading of liquids. Langmuir 32, 10153-10158.

Karim, A. M., J. P. Rothstein and H. P. Kavehpour (2018). Experimental study of dynamic contact angles on rough hydrophobic surfaces. Journal of Colloid and Interface Science 518, 658-665.
Karim, A. M., K. Fujii and H. P. Kavehpour (2021). Contact line dynamics of gravity driven spreading of liquids. Fluid Dynamics Research 53, 035503.

Petrov, P. G. and J. G. Petrov (1992). A combined molecular-hydrodynamic approach to wetting kinetics. Langmuir 8, 1762-1767.

Pinkus, O. and B. Sternlicht (1961). Theory of hydrodynamic lubrication. McGraw-Hill, New York.

Yen, T. H., C. Y. Soong and P. Y. Tzeng (2007). Hybrid molecular dynamics-continuum simulation for nano/mesoscale channel flows. Microfluidics and Nanofluidics 3, 665-675.

Zhang, Y. B., K. Tang and G. S. Lu (2003). Model of elastohydrodynamic lubrication with molecularly thin lubricating films: Part IDevelopment of analysis. International Journal of Fluid Mechanics Research 30, 542557.

Zhang, Y. B. and G. S. Lu (2003). Model of elastohydrodynamic lubrication with molecularly thin lubricating films: Part IIResults for an exemplary lubrication. International Journal of Fluid Mechanics Research 30, 558-571.

Zhang, Y. B. (2016). The flow equation for a nanoscale fluid flow. International Journal of Heat and Mass Transfer 92, 1004-1008.

Zhang, Y. B. (2020). Modeling of flow in a very small surface separation. Applied Mathematical Modelling 82, 573-586.

Zhang, Y. B. (2021a). Multiscale mixed hydrodynamics in line contacts, Continuum Mechanics and Thermodynamics, https://doi.org/10.1007/s00161-021-01068-2.

Zhang, Y. B. (2021b). Multiscale hydrodynamics in line contacts. Mechanics Research Communications 111, 103658. 